\title{
Study on Operation Proficiency of Surgery Robot User
}

\author{
Yan Zhou ${ }^{1, a}$, Chunyang Wang ${ }^{2, b}$, and Lei Zhü ${ }^{3, c}$ \\ ${ }^{1}$ School of xxx, xxx University, Guangzhou 510000, China; \\ ${ }^{2}$ School of yyy, zzz University, Guangzhou 510000, China. \\ ${ }^{1}$ School of Mechatronic Engineering, Harbin Institute of Technology, Harbin, 150001, China; \\ ${ }^{2}$ School of Mechatronic Engineering, Harbin Institute of Technology, Harbin, 150001, China; \\ ${ }^{3}$ School of Mechatronic Engineering, Harbin Institute of Technology, Harbin, 150001, China; \\ ahitdesign@163.com, bwspringy@icloud.com, leizcn@163.com
}

Keywords: Surgery Robots, Operation Proficiency, Analytic Hierarchy Process, Combination of Subjective and Objective Realities, Assessment Model.

\begin{abstract}
With the rapid technological development of surgery robots nowadays, however, there is not scientific assessment system to accurately and objectively study on operation proficiency of surgery robot user. A scientific and precise comprehensive assessment model is established on a scientific assessment indicator system, with the scientific assessment indicator system, the comprehensive assessment results can be obtained. The paper aims to elaborate how to establish a scientific assessment model to evaluate the proficiency of primary surgical operation by robot. Here is the analysis method: the preliminary assessment indicators about the proficiency of primary surgical operation by robot are widely researched and collected by means of expert opinions and literature review, and then with the analytic hierarchy process, the assessment indicators will be further selected to ensure the weight of each grade of assessment indicators, and finally to establish an optional assessment indicator system.
\end{abstract}

\section{Introduction}

The technology of surgery robots, as a typical representative of medical instrument with high technology, is an interdisciplinary research incorporated with medical science, mechanics, robotics, computer graphics, computer vision and mathematical analysis. The technology can accomplish or assist in accomplishing the complex surgery treatment that cannot be done with various conventional instruments. Compared with traditional surgery, the robotic surgery can better the patient's life for its advantages of small wound, little adhesion, recovery time reduction after the surgery and suffering alleviation $^{[1]}$.

During the process of practical surgery operation, surgeons accomplish a series of operation tasks with the skilled hand, therefore, with the different proficiency of the surgeon skilled hand, the operation will be influenced. However, the assessment of operation proficiency towards new doctors is not established with the industry standard ${ }^{[2]}$, and the judgement is only based on specialists, thus lacking an objective and precise assessment method ${ }^{[3]}$. With the analytic hierarchy process and fuzzy evaluation method, the paper aims to establish an assessment model with objective and subjective ${ }^{[4]}$ consideration so as to realize the objective and precise assessment of the proficiency of surgical operation by robot's skilled hand.

\section{Establish the Assessment System}

\subsection{Preliminary Proposals and Hierarchical Structure of the Assessment System}

A scientific and precise comprehensive assessment model is established on a scientific assessment indicator system, and before establishing the assessment system, assessment indicators should be selected in the principle of completeness, scientific independence, operability, hierarchical structure 
and its purpose. After the analysis of relevant documents, it is found out that nowadays the assessment operation proficiency of surgery robot operator is mostly based on the subjective judgement of specialists, yet some practitioners have quantitative and objective judgement through experiment design. In the paper, with the subjective and objective considerations, the assessment indicators are established in the perspectives of subjective assessment elements and objective assessment elements.

The preliminary assessment indicators about the proficiency of primary surgical operation by robot are widely researched and collected by means of expert opinions and literature review. With the experience of laparoscopic operation, a group of 8 specialists are selected for objective judgement. The emails and interview consultations, with contents of the preference of assessment indicators and raising questions with hierarchical structure, are sent to 8 specialists who do not meet with each other, and then different opinions will be collected. Each time of asking and answering one question is called the first round. The information of each specialist's different opinions and the degree of conformity in the first round is then reflected to each specialist in the second round. In this way, three rounds are performed, and then the opinions of specialists will basically reach to a consensus.

At last the preliminary assessment indicators of listing and hierarchical structure is acquired. Here are the first grade assessment indicators: assessment of motion tracking metrics--B1 ${ }^{[5]}$, assessment of task completion--B2, assessment of physiological performance--B3. The second grade assessment indicators affiliated with assessment of motion tracking metrics--B1 are: time of task completion, total travel distance, average speed, number of mistakes; the second grade assessment indicators affiliated with assessment of task completion--B2 are: operation accuracy, operation order, operation stability, operation agility, operation simplicity; the second grade assessment indicators affiliated with assessment of physiological performance--B3 are: degree of relaxation of upper limb, degree of relaxation of lower limb, degree of posture standard, degree of relaxed look ${ }^{[6]}$.

\subsection{Screening of Assessment Indicators.}

With the analytic hierarchy process, the assessment indicators of preliminarily hierarchical structure can be further screened. Based on the definition table of judgement matrix method of nine scales and with the help of questionnaire, the 8 specialists respectively compare and give marks for two of any three first grade assessment indicators, including assessment of motion tracking metrics--B1, assessment of task completion--B2, assessment of physiological performance--B3.

Each achieved judgement matrix can be made the conformity test with the application of random consistency ratio C.R. If C.R. $<0.10$, the conformity of judgement matrix is acceptable; if C.R. $\geqslant 0.10$, the conformity of judgement matrix is unacceptable. After meeting the criterion of conformity, each group of judgement matrix can be integrated into the comprehensive judgement matrix through the weighted average, and then to test the conformity test of comprehensive judgement matrix, and finally to test the conformity of comprehensive judgement matrix of motion tracking metrics, task completion and physiological performance.

Please refer to the above screening method for the second grade assessment indicators affiliated to the first grade assessment indicators.

\subsection{Verify the Final Structure of Evaluation System}

The eigenvalues of judgement matrix and its corresponding eigenvector can be calculated by MATLAB software. After the uniformity of the eigenvector, the achieved vector is the weight vector of assessment indicators. With the above method of weight calculation, the weight of three groups of the second grade assessment indicators can be obtained. For the two weights of muscle activity factor and operation coordination factor are less than 0.05, the final assessment structure system can be acquired excluding this two indicators. 


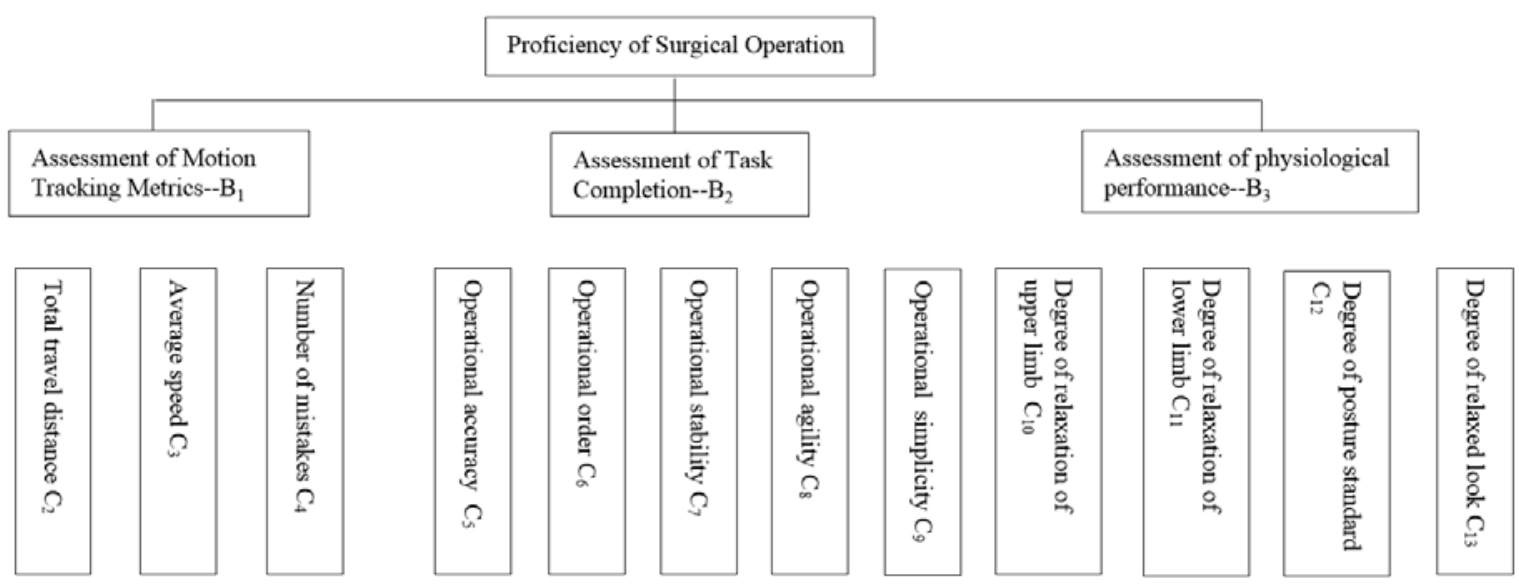

Fig. 1 Final Structure of Evaluation System

\section{Ensure the Weight of Each Grade Assessment Indicator}

The second grade assessment indicators to the weight of their respectively affiliated first grade assessment indicators can be confirmed with another uniformity of the after-screening second grade assessment indicators. The weight calculating method of the first grade assessment indicator is the same with that of the second grade assessment indicator. The above calculating method can finally ensure the weight of each grade of assessment indicators.

Table 1 Weight Diagram of Each Grade of Assessment Indicators

\begin{tabular}{cccc}
\hline Primary index & weight & Secondary index & weight \\
\hline $\mathrm{B}_{1}$ & 0.3261 & $\mathrm{C}_{1}$ & 0.2067 \\
& & $\mathrm{C}_{2}$ & 0.0774 \\
& & $\mathrm{C}_{3}$ & 0.1519 \\
$\mathrm{~B}_{2}$ & \multirow{2}{*}{0.6040} & $\mathrm{C}_{4}$ & 0.5639 \\
& & $\mathrm{C}_{5}$ & 0.4821 \\
& & $\mathrm{C}_{6}$ & 0.1042 \\
& $\mathrm{C}_{7}$ & 0.2309 \\
$\mathrm{~B}_{3}$ & & $\mathrm{C}_{8}$ & 0.1036 \\
& \multirow{3}{*}{0.0699} & $\mathrm{C}_{9}$ & 0.0791 \\
& & $\mathrm{C}_{10}$ & 0.5262 \\
& & $\mathrm{C}_{11}$ & 0.2051 \\
& & $\mathrm{C}_{12}$ & 0.0703 \\
& & $\mathrm{C}_{13}$ & 0.1984 \\
\hline
\end{tabular}

\section{Qualitative and Quantitative Assessment Methods}

\subsection{Preliminary Proposals and Hierarchical Structure of the Assessment System}

The test subjects, with the average age of $24 \pm 2$ and right-handed habit, is made of 15 college students ( 8 boys and 7 girls) from Harbin Institute of Technology. Before the test, all of them have no experience of skilled hand operation of surgery robots.

The instruments adopted in the test are the operation platform of virtual reality and Omega.7.t with skilled hand instrument from Force Dimension company, and the test subject finishes the experiment task in the virtual platform through operating the skilled hand device. Small bowls in red, green and blue and 6 small balls in red, green and blue are set in the virtual platform. By operating the skilled hand instrument of Omega. 7, the test subject clamps the small ball in different color to the respective bowl. The figures tested in the motion tracking metrics experiment are comprised of time of task completion, total travel distance of pinpoint, average speed and number of mistakes ${ }^{[7]}$. 


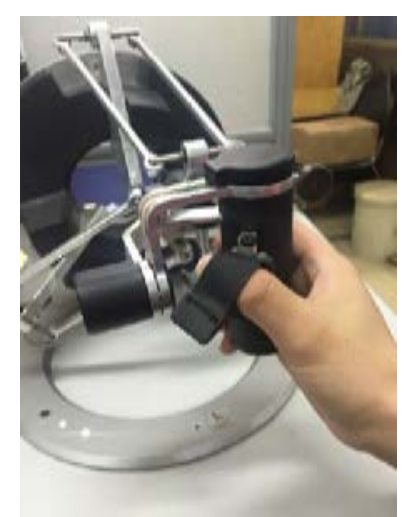

Fig. 2 Omega.7.t skilled hand

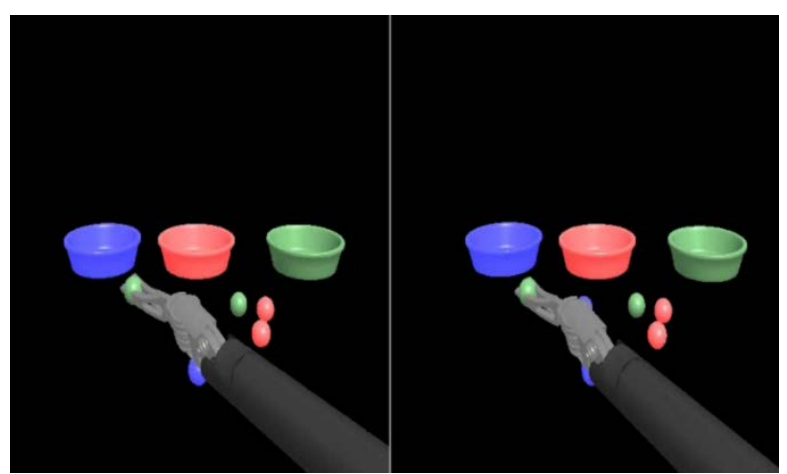

Fig. 3 Interface of virtue platform experiment

There are three phases in the experiment:

Phase 1: Firstly, the tester has a basic operation training for the test subjects, illustrating in detail the instruction, experiment procedure and notices of experiment instrument. After being familiar with the experiment task, the test experiment begins and the tester records the test figures in detail.

Phase 2: After the phase 1, each test subject has one hour for rest, and after the rest each test subject has one hour of practice, during the practice, the test subject can ask any question of experiment operation and the tester answers the question in detail.

Phase 3: Phase 3 has the same procedure with phase 2, after finishing the 3 phases, the task of experiment is over.

\subsection{Assessment Method of Specialists}

Based on the performance of test subjects in the third motion tracking metrics experiment, 8 specialists judges each test subject from the operation performance of qualitative indicator assessment to give a score in the 4 levels of being excellent, good, qualified and unqualified.

\section{Model Construction of Fussy Comprehensive Assessment}

\subsection{The Confirmation in Degree of Membership of Qualitative Assessment Indicators}

Through collecting specialists' scoring result for each test subject i the operation performance of second degree qualitative indicator assessment, the degree of membership is confirmed with rating proportioning method, that is, the degree of membership ${ }^{[8]}$ of each factor affiliated to 4 respective levels is each factor affiliated to the number of 4 levels respectively divided by the number of specialists.

For example, for the operation accuracy of the qualitative assessment indicator C5, the 8 specialists give a respective score with the standard of being excellent, good, qualified and unqualified, for the first test subject based on the operation performance. After collecting the 8 results from specialist, it is found that the first test subject wins 6 excellent results, 2 good results, 0 qualified result and 0

unqualified result. Therefore, in the test subject's assessment indicator of operation accuracy, the degree of membership for being excellent is $75 \%$, the degree of membership for being good is $25 \%$ and the degree of membership for being qualified and unqualified is 0 . Based on the third operations of performance of test subjects, the specialists can get the degree of membership of second qualitative assessment indicator to the scoring level. The degree of membership of first grade qualitative assessment indicator can be acquired based on the weight of each second grade assessment indicator.

\subsection{Confirmation in Degree of Membership of Qualitative Assessment Indicators}

The second grade assessment indicators are: $\mathrm{C}_{1}$ time of task completion, $\mathrm{C}_{2}$ total travel distance, $\mathrm{C}_{3}$ average speed, $\mathrm{C}_{4}$ number of mistakes; Based on the qualitative result from the experiment of motion tracking metrics and specialist assessment method, the qualitative result can be mapped to the interval of $[0,1]$ to get the degree of membership of qualitative assessment indicators.

Here is the piecewise function of $\mathrm{C}_{2}$ task completion:

The degree of membership of being excellent is: The degree of membership of being good is: 


$$
y_{1-1}(x)=\left\{\begin{array}{c}
1,0 \leq \mathrm{x}<25 \\
\frac{50-x}{25}, 25 \leq x<50 \\
0, \text { others }
\end{array}\right.
$$

The degree of membership of being qualified is: The degree of membership of being unqualified is:

$$
y_{1-3}(x)=\left\{\begin{array}{cc}
\frac{x-50}{25}, & 50 \leq \mathrm{x}<75 \\
\frac{100-x}{25}, & 75 \leq x<100 \\
0, & \text { others }
\end{array}\right.
$$

$$
y_{1-4}(\mathrm{x})=\left\{\begin{array}{c}
\frac{x-75}{25}, 75 \leq x<100 \\
1, \mathrm{x} \geq 100 \\
0, \text { others }
\end{array}\right.
$$

Here is the piecewise function of $\mathrm{C}_{2}$ pinpoint total travel distance:

The degree of membership of being excellent is: The degree of membership of being good is:

$$
y_{2-1}(\mathrm{x})=\left\{\begin{array}{c}
\frac{x-15}{5}, 15 \leq x<20 \\
1, \mathrm{x} \geq 20 \\
0, \text { others }
\end{array}\right.
$$

$$
y_{2-2}(\mathrm{x})=\left\{\begin{array}{cc}
\frac{x-10}{25}, & 10 \leq \mathrm{x}<15 \\
\frac{20-x}{5}, & 15 \leq x<20 \\
0, & \text { others }
\end{array}\right.
$$

The degree of membership of being qualified is: The degree of membership of being qualified is:

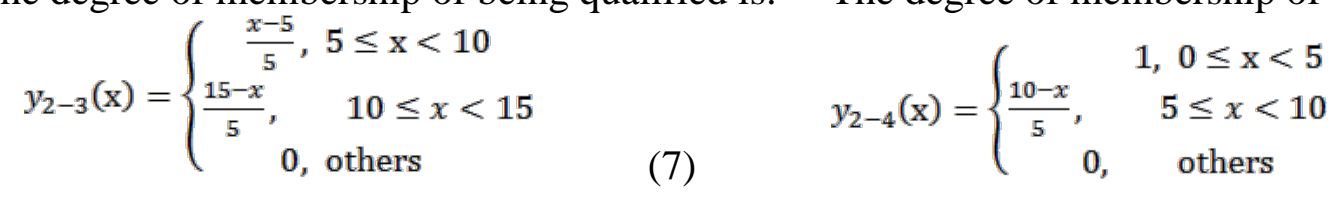

C3 average speed and C1 task completion have the same piecewise function.

Here is the piecewise function of $\mathrm{C} 4$ number of mistakes:

The degree of membership of being excellent is: The degree of membership of being good is

$$
y_{4-1}(x)=\left\{\begin{array}{cc}
1, & 0 \leq x<0.5 \\
1.5-x, & 0.5 \leq x<1.5 \\
0, & \text { others }
\end{array}\right.
$$

$$
y_{4-2}(x)=\left\{\begin{array}{c}
x-0.5,0.5 \leq x<1.5 \\
2.5-x, 1.5 \leq x<2.5 \\
0, \text { others }
\end{array}\right.
$$

The degree of membership of being qualified is: The degree of membership of being unqualified is:

$$
y_{4-3}(\mathrm{x})\left\{\begin{array}{c}
\mathrm{x}-1.5,1.5 \leq \mathrm{x}<2.5 \\
3.5-x, 2.5 \leq x<3.5 \\
0, \text { others }
\end{array}\right.
$$

$$
y_{4-4}(x)=\left\{\begin{array}{c}
x-2.5,2.5 \leq x<3.5 \\
1, x \geq 3.5 \\
0, \text { others }
\end{array}\right.
$$

In the test subject's experiment of motion tracking metrics, the test figures of time of task completion, pinpoint total travel distance, average speed and number of mistakes are input to the piecewise function affiliated to each assessment indicator to get the degree of membership in the second degree qualitative assessment indicators, and then the degree of membership of first grade assessment indicator motion tracking metrics can be acquired based on the weight of assessment indicators.

\subsection{Transfer the Score of Each Test Subject to the Centesimal System}

the score of each test subject is transferred to the form of centesimal system, in the formula of (13) Q is the general score, $\mathrm{B}$ is the uniformity result of $\mathrm{B}$ with the first hierarchy assessment, and $\mathrm{C}$ is $\quad[92.5$ $\left.\begin{array}{lll}77.5 & 65 & 30\end{array}\right]$.

$$
\mathrm{Q}=B^{r} \cdot C^{T}
$$

\section{Credibility Inspection of Comprehensive Assessment Model}

In the study, Cronbach ${ }^{\wedge} \alpha$ coefficient is used to test the credibility of comprehensive assessment model. After the analysis Cronbach ${ }^{\wedge} \alpha$ coefficient of assessment model in this study is more than 0.9 , which means that the assessment model passes the credibility inspection with a very good credibility ${ }^{[9]}$. 


\section{Credibility Inspection of Comprehensive Assessment Model}

In order to realize the objective and precise assessment of operation proficiency for surgery robot users, the paper adopts the qualitative and quantitative assessment methods. With the specialist assessment method and literature analysis method, the assessment indicators are widely collected through the skilled hand operation proficiency of the surgery robot. Analytic hierarchy process is applied to screen the assessment indicators and to confirm the weight of each grade assessment indicators, and then to construct the model of fussy comprehensive model. After the credibility inspection of assessment model, operation proficiency for surgery robot user can be assessed with the constructed comprehensive model ${ }^{[11]}$.

\section{References}

[1] Chapman WH III, Albrecht RJ, Kim VB, Young JA, Chitwood WR Jr (2002) Computer-assisted laparoscopic splenectomy with the da Vinci surgical robot. J Laparoendosc Adv Surg Tech A 12:155-159.

[2] Elliot DL, Hickam DH. Evaluation of physical examination skills. Reliability of faculty observers and patient instructors. JAMA 1987; 258:3405-8.

[3] Warf BC, Donnelly MB, Schwartz RW, Sloan DA. Interpreting the judgment of surgical faculty regarding resident competence. J Surg Res 1999;86:29-35.

[4] REILEY Carol E, LIN Henry C, YUH David D, et al. Review of methods for objective surgical skill evaluation[J]. Surgical Endoscopy, 2011.

[5] Rosser JC, Rosser LE, Savalgi RS. Skill acquisition and assessment for laparoscopic surgery. Arch Surg 1997;132:200-4.

[6] Scott DJ, Bergen PC, Rege RV, et al. Laparoscopic training on bench models: better and more cost effective than operating room experience? J Am Coll Surg 2000;191:272-83.

[7] Abbas F, Coburn M. Education and Training of an Academic Urologic Surgeon[J]. Urolithiasis, 2012.

[8] Wang YuJie. A fuzzy multi-criteria decision-making model by associating technique for order preference by similarity to ideal solution with relative preference relation[J]. Information Sciences, 2014,268:169-184

[9] CHEN S M. Fuzzy group decision making for evaluating

the rate of aggregative risk in software development [ $\mathrm{J}$ ].

Elsevier Science, 1997 (3) : 17228.

[10] Lu Zi, Wang Sisi, Li Xiaonan, Yang Lihua, Yang Dong, Wu Dianshuang. Online shop location optimization using a fuzzy multi-criteria decision model - Case study on Taobao.com[J]. Knowledge-Based Systems, 2012,32:76-83. 